\title{
The queue length estimation for congested signalized intersections based on shockwave theory
}

\author{
Yu Li, Dan-pu Zhao \\ Institute of Automation, Chinese Academy of Science \\ $\mathrm{Wu} \mathrm{Xi}$, China \\ constance.lee1008@gmail.com
}

\begin{abstract}
The queue length at signalized intersection is critical to either signal performance measure in terms of vehicle delay and stops or signal optimization. The traditional deterministic queuing theory based on the hypothesis that the flow of the road is steady during the entire time which disagrees with the actual situation. In this paper, instead of instead of using the deterministic queuing theory we solve the problem by modeling the queue dynamics in the signalized intersection with the Lighthill-Whitham-Richards(LWR) shockwave theory. We use the detector to identify traffic state changes by analysis the data in the immediate past cycle collected by the detector settled in the road. When the queue length is longer than the detector we can still distinguish the queue discharge flow from upstream arrival traffic. Therefore our approach can estimate queue length of the saturated Intersections. We used VISSIM to comparing our model with the SIGNAL94 model and evaluated by comparing the estimate maximum queue length with the queue length outputted by VISSIM. The results demonstrate that the proposed model can estimate long queues with satisfactory accuracy.
\end{abstract}

\section{Index Terms-Queue length, LWR, VISSIM, Shockwave}

\section{INTRODUCTION}

Vehicular queue length and queuing duration are important basis for traffic management departments to establish and implement traffic control measures. The study of the vehicular queue length and queuing duration has important practical significance and value.

Jian Rong, Man Ho [1] compared varieties of domestic and foreign queuing models and according to the actual situation of Beijing, established a dynamic calculation queuing model on the basis of the measured data of Beijing. Xue-nong Zhou [2] analysis the results of multiple classic queuing models by means of the measured data of Changsha. By contrasting the results, he found out the most appropriate queue length optimization model. Lei-lei Dai, Gui-yan Jiang [3] predicted the real time traffic flow at entrance lane and established the queue length prediction model on the basis of the deterministic queuing theory and validated the result by experiments. Deterministic queuing theory is the traditional queuing theory for the saturated traffic situation. However, this theory is based on the premise that the average vehicles arrival rate at entrance lane is stable throughout the period of time, which does not comply with the actual situation. In this paper, we provide the model with the traffic wave theory, which is adapted to the saturate traffic situation and used VISSIM simulation software to validate and compare the results.

\author{
Xian-qing Tai, Zhi-li Liu \\ Institute of Automation, Chinese Academy of Science \\ Wu Xi, China \\ xianqing.tai@ia.ac.cn
}

\section{SHOCK WAVE ANALYSIS}

The traffic flow theory was first demonstrated by Lighthill and Whitham and Richards. The traditional LighthillWhithan-Richatds (LWR) model hypothesizes that flow is a function of density at any point of the road. Traffic shockwave theory is derived from LWR model and it depicts the motion of an abrupt change in concentration[4-7].

The establishment of the basic model of the traffic shockwave is shown in Fig1. We hypothesis that there are two adjacent regions with different density on the road and the vertical line $\mathrm{S}$ separate the two regions. We hypothesis the velocity of $\mathrm{S}$ is $u_{w}$. $u_{w}$ can be determined by following equation[8-9]:

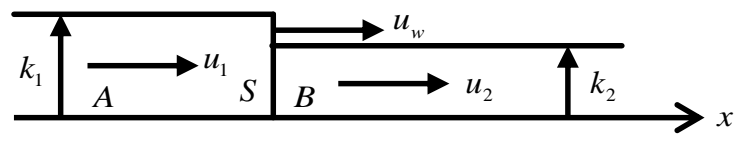

Fig. 1. the operation of two kinds of flow densities

$$
u_{w}=\left(q_{2}-q_{1}\right) /\left(k_{2}-k_{1}\right)
$$

Where $q_{1}, k_{1}, u_{1}$ are the flow, density and velocity of the A region and $q_{2}, k_{2}, u_{2}$ are the flow, density and velocity of the $\mathrm{B}$ region.

We simply assume that queue has been fully discharged during the last green phase. In the following red interval, vehicles are forced to stop. Such interruption of traffic flow forms a queuing shockwave $v_{l}$ in Fig. 1 moving upstream of the intersection with velocity

$$
v_{l}=\left(0-q_{a}^{n}\right) /\left(k_{j}-k_{a}^{n}\right)
$$

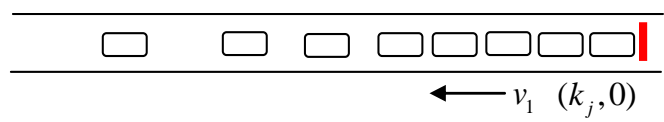

Fig. 2. Shock wave $v_{1}$ propagation

where 0 and $k_{j}$ represent the jammed flow and density; $q_{a}{ }^{n}$ and $k_{a}{ }^{n}$ are the average arrival flow rates and density during the $n$th cycle. 
At the beginning of the effective green, vehicles begin to discharge at saturation flow rate forming the second shock wave which is defined as discharge shockwave $v_{2}$ at the stop line moving upstream with speed

$$
v_{2}=\left(q_{m}-0\right) /\left(k_{m}-k_{j}\right)
$$

where $q_{m}$ and $\mathrm{km}$ are the capacity or saturation flow and density.

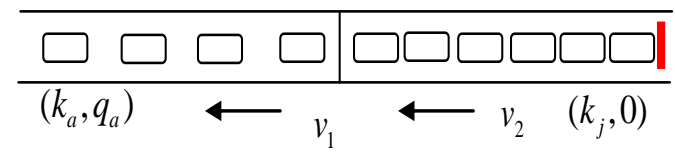

Fig. 3. Shock wave $v_{2}$ propagation

The discharge shockwave $v_{2}$ usually has higher speed than $v_{l}$, so the two waves will meet at time $T_{\max }$, which is the time that this approach has the maximum queue length. As soon as the two shock waves meet, a third one $v_{3}$ is generated propagating toward the stop line with speed

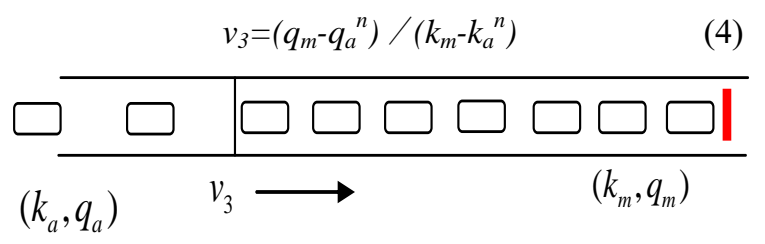

Fig. 4. Shock wave $v_{3}$ propagation

Traffic shockwave can be also illustrated by using the fundamental diagram ( $q-k$ curve).

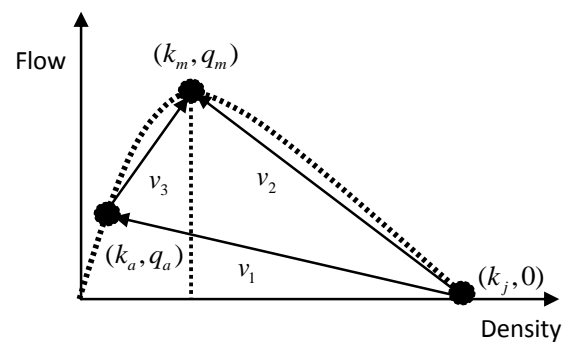

Fig. 5. Representation of shockwaves in the fundamental diagram

\section{BREAKPOINT IDENTIFICATION}

We use the detectors to detect the time point $A, B$ and $C$, which represent the time instants that traffic condition changes within a cycle. In detail, the time that point $A$ appears $\left(T_{a}\right)$ is the moment that the queuing shock wave $V_{l}$ propagates backward to the location of the detector. Between the end of green in the nth cycle and $T_{a}$, the vehicles pass the loop detector with the traffic state $\left(k_{a}, q_{a}\right)$; while between $T_{a}$ and the time of maximum queue achieved, no vehicle can pass the loop detector because of the jam traffic condition $\left(k_{j}, 0\right)$. Point $A$ can be used to judge whether there is a long queue or not, as after $T_{a}$, the detector is occupied for a relatively long time, so the value of the detector occupancy time is relative large. A threshold value is necessary for practical application. In this study, based on our observation, $4 s$ is a large enough number to check whether point $A$ exists. If the detector occupancy time is larger than $4 s$, the intersection has long queue; and vice versa. We should point out that second-bysecond percentage occupancy data can also be utilized to identify point $A$, i.e. the occupancy value is kept at $100 \%$ for more than $3 s$.

Point $B$ indicates the time $\left(T_{b}\right)$ that the discharge shockwave passes the detector. Between effective green start and $T_{b}$ the traffic state over the detector is $\left(k_{j}, 0\right)$; after $T_{b}$, vehicles are discharged at saturation flow rate and traffic state changes to $\left(k_{m}, q_{m}\right)$. After the green starts and before $T_{b}$, traffic volume is zero, and detector occupancy time is high (larger than $4 s$ ) or second-by-second percentage occupancy continues to be $100 \%$ for at least $4 s$. After $T_{b}$, queued vehicles begin to discharge over the detector, therefore both detector occupancy time and time gap between consecutive vehicles drop.

Point $C$ indicates the time $\left(T_{c}\right)$ when the rear end of queue passes the detector. As introduced before, wave $v_{3}$ is the interface between saturation traffic state $\left(k_{m}, q_{m}\right)$ and the arrival traffic state $\left(k_{a}, q_{a}\right)$. Therefore, before point $C$ appears, vehicles discharge at the saturation flow rate at the location of loop detector, the traffic state is $\left(k_{m}, q_{m}\right)$ After the wave propagates to the detector location, the traffic condition becomes to $\left(k_{a}, q_{a}\right)$, the discharge rate at the loop detector location is less than saturation flow. A threshold should be selected to identify the two different traffic states $\left(k_{m}, q_{m}\right)$ and $\left(k_{a}, q_{a}\right)$. Based on our observation, after $T_{c}$, the vehicle gaps become much bigger and the variance is significantly increased.

Considering the variation of time gaps, using a single value to separate traffic states may bring large error. In our implementation, if the time gap is between $2 s$ and $3 s$, which means $0 \%$ occupancy for at least two consecutive seconds, the system will continue searching the second and third points with time gaps over $2 s$ to make sure that the traffic state is really changed.

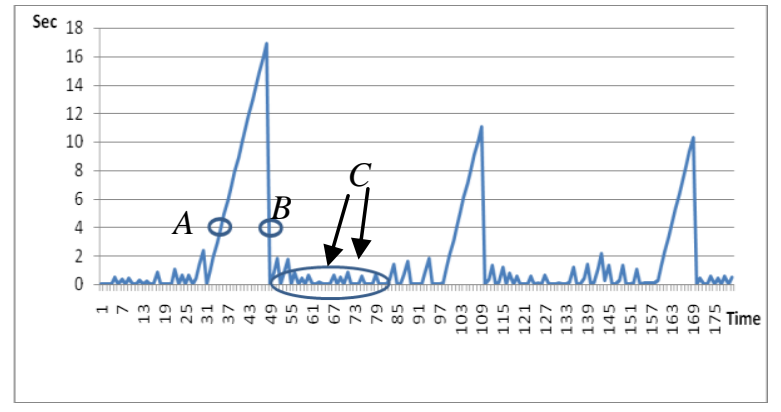

Fig. 6. Time gap between consecutive vehicles 


\section{THE ESTABLISHMENT OF MODEL}

The models proposed here are to utilize points $A, B$ and $C$ identified in the last section using detector.

As mentioned above, wave $v_{2}$ traveled the same distance with wave $v_{3}$, so we can come to the conclusion that

Finally, complete content and organizational editing before formatting. Please take note of the following items when proofreading spelling and grammar.

$$
\begin{aligned}
L_{\text {max }} & =v_{2} \times\left(T_{\max }-T_{b}\right) \\
L_{\text {max }} & =v_{3} \times\left(T_{c}-T_{\text {max }}\right) \\
L_{\text {max }} & =L_{d}+L_{e x}
\end{aligned}
$$

where $L_{d}$ is the distance from stop line to the loop detector, lexis the distance from the detector to the rear end of the queue.

Combine the three equation above, considering the influencing factors of the complicated traffic environment, the queue length model is that

$$
L_{\max }{ }^{n}=L_{d}+\alpha \times\left(\left(T_{c}-T_{b}\right) /\left(1 / v_{2}+1 / v_{3}\right)\right.
$$

Where $\alpha$ is the correction coefficient.

\section{IMPLEMENTATION}

We use VISSIM to verify the model. We use single lane with the lane width of $3.5 \mathrm{~m}$ and the intersection signal cycle was $60 \mathrm{~s}$ with the green light cycle for 30 s. The detector is placed at a distance of the intersection of $100 \mathrm{~m}$. The source of the traffic is $600(v e h / h)$, and increase $600(v e h / h)$ every 5 cycles. When the flow reaches to $1200(v e h / h)$, after 10 cycles, the source of the traffic decrease 200 (veh/h) every 5 cycles to $600(\mathrm{veh} / \mathrm{h})$. We use the default setting of VISSIM, which assume that when the velocity of the car is less than $5 \mathrm{~km} / \mathrm{h}$, the car is in queuing state. In VISSIM, the saturation density $k_{j}=130(\mathrm{veh} / \mathrm{h})$ and the saturation flow is $1800(\mathrm{veh} / \mathrm{h})$. We can know from Grenberg model that

$$
k_{m}=k_{j} / e
$$

We can know from the equation that $k_{m}=48.1(\mathrm{veh} / \mathrm{km})$, and because

We can know that

$$
q=k \times v
$$

$$
v_{2}=37.4(\mathrm{~km} / \mathrm{h})
$$

Occupancy time recorded by detectors can be used to estimate the density. Eq. (14) is used to estimate the density $(k)$ ( ignore the length of detector)

$$
\begin{gathered}
o=\frac{\sum_{i}\left(l_{i}+d\right) / u_{i}}{T}=\frac{1}{T} \sum_{i} \frac{l_{i}}{u_{i}}+\frac{d}{T} \sum_{i} \frac{1}{u_{i}} \\
o=\left(\frac{1}{N} \sum_{i} \frac{l_{i}}{u_{i}}\right) / \bar{h}+d k=l_{i} \frac{q}{\bar{u}_{s}}+d k=\left(l_{i}+d\right) k=l_{i} k
\end{gathered}
$$

We can know that:

$\begin{array}{ll}k=o / l_{i}=\left(\frac{1}{T} \sum_{i} \frac{l_{i}}{u_{i}}\right) / l_{i} & (14) \\ \text { Where } k \text { is de } & \text { pancy }(\%) ; I \text { is }\end{array}$ the length of the $i$-th vehicle $(m), \mathrm{d}$ is the length of the detector $(m) ; u_{i}$ is the velocity of the $i$-th vehicle $(\mathrm{km} / \mathrm{h})$; $\mathrm{T}$ is the observation time $(h)$ 。

We utilize least square method to determine $\alpha$ is 1.25

We use the maximum queue to contrasted SIGNAL94 model with our approach. The results of the maximum queue length

\begin{tabular}{|c|c|c|c|}
\hline \multirow[t]{2}{*}{ Cycle } & \multicolumn{3}{|c|}{ The queue length of the cars } \\
\hline & The real queue length $(\mathrm{m})$ & $\begin{array}{l}\text { The shock wave } \\
\text { model }(\%)\end{array}$ & SIGNAL94(\%) \\
\hline 1 & 138.6300105 & 27.41667111 & 16.58997737 \\
\hline 2 & 178.0004038 & 8.048454461 & 32.64280619 \\
\hline 3 & 170.6227949 & 9.689174435 & 44.41266992 \\
\hline 4 & 166.0807532 & -6.205744543 & 44.35571734 \\
\hline 5 & 157.9959744 & 8.284763462 & 27.86344065 \\
\hline 6 & 122.133828 & 9.702974769 & 11.07130227 \\
\hline 7 & 125.6378151 & 7.101553623 & 11.06264746 \\
\hline 8 & 133.5930752 & 12.07489446 & 7.835527851 \\
\hline 9 & 140.3550032 & 8.69990055 & 21.06245646 \\
\hline 10 & 151.840407 & 10.92510401 & 14.40286836 \\
\hline 11 & 169.2323734 & 4.825467543 & 45.45221039 \\
\hline 12 & 113.7971302 & 10.55850713 & 4.354543495 \\
\hline 13 & 133.1725799 & 8.522951733 & 12.9924935 \\
\hline 14 & 181.9414448 & 7.538662104 & 53.39141009 \\
\hline 15 & 254.160265 & 10.22029311 & 130.53772 \\
\hline 16 & 212.2348724 & 10.49534911 & 82.05731182 \\
\hline 17 & 221.9211904 & 10.96112522 & 88.50354569 \\
\hline 18 & 169.2323734 & 7.587225646 & 30.57993952 \\
\hline 19 & 140.3550032 & 9.713574895 & 18.78624686 \\
\hline
\end{tabular}
are presented in Fig. 7; and Table 1 shows the Mean Absolute Relative Error (MARE) which is calculated by

$$
M A R E=\frac{1}{N} \sum_{i=1}^{N}\left|\frac{y_{i}(t)-y_{i}(t)}{y_{i}(t)}\right|
$$

The traffic flow is instability at the initial of the simulation and the flow failed to reach the saturation point, therefore we took the data when the traffic state is saturated.

TABLE I. MAXIMUM QUEUE LENGTH COMPARISON 


\begin{tabular}{|c|c|c|c|}
\hline Cycle & \multicolumn{3}{|c|}{ The queue length of the cars } \\
\hline & The real queue length(m) & $\begin{array}{c}\text { The shock wave } \\
\text { model(\%) }\end{array}$ & SIGNAL94(\%) \\
\hline 20 & 151.840407 & 7.202794304 & 29.30185917 \\
\hline 21 & 115.6378151 & 9.361861751 & 12.65218328 \\
\hline 22 & 133.5930752 & 11.46617072 & 21.13621131 \\
\hline
\end{tabular}

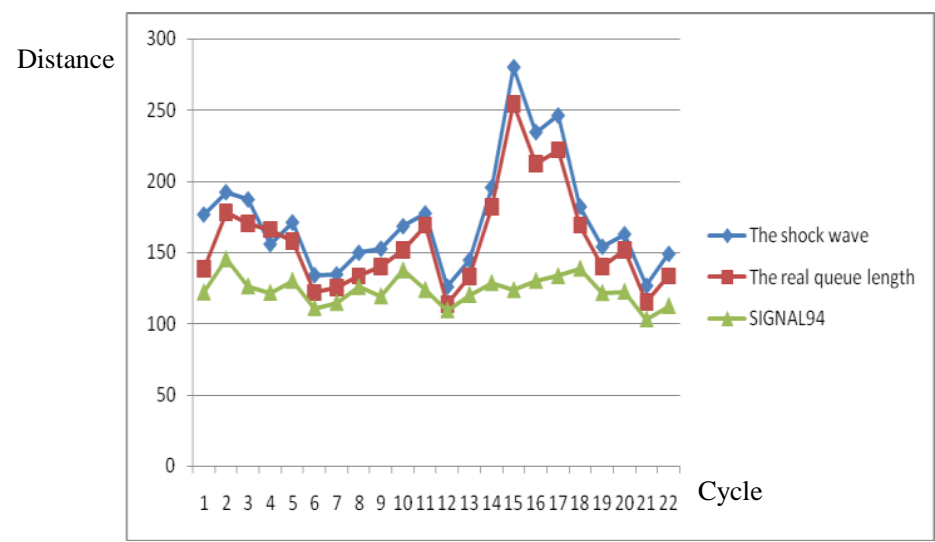

Fig. 7. Maximum queue length comparison

From the results, we can see that the maximum absolute error of the traffic wave model is $38.008 \mathrm{~m}$ and the average absolute error is $14.488 \mathrm{~m}$; the maximum relative error is $27.417 \%$; the mean relative error of $9.281 \%$ (up to $90 \%$ accuracy); the maximum absolute error of SIGNAL94 queuing model based on a set number theory is $130.538 \mathrm{~m}$, the mean absolute error is $34.593 \mathrm{~m}$, the maximum relative error is $51.36 \%$, the average relative error is $19.367 \%$, therefore, we can conclude that the revised traffic flow model in this paper performed better than SIGNAL94 model under the condition that the traffic flow is unsteady and saturated.

\section{COPYRIGHT FORMS}

In this paper, we analyzed the propagation of the shockwave, and established a model that can estimate the real-time queue length at the congested signalized intersection. And we testified the result by established the model with the VISSIM simulation software, and contrast the result with the traditional deterministic queuing theory. The results showed that, the model we established with the shockwave theory has a better accuracy than the deterministic queuing theory, especially when the queue length exceed the distance between the detector and the stop line. And the accuracy can reach to $90 \%$, which can satisfy the demand of traffic control and help the authorities to make policy.

\section{ACKNOWLEDGMENT}

The authors would like to thank to the anonymous reviewers of this paper for the constructive comments.

\section{REFERENCES}

[1] Jian Rong, Min He, Chun-mei Chen. Dynamic model of calculating queue length at signalized intersection .China Journal of Highway And Transport, 2002,15(3), pp.101-104

[2] Xue-nong Zhou. Queue length model comparison and dynamic modeling .Transportation Systems Engineering and Information, 2006, 6(1), pp.91-95

[3] Lei-lei Dai, Gui-yan Jiang, Yulong Pei. Prediction of queue length at saturate signalized intersection [J]. Journal of Jilin University (Engineering and Technology Edition). Nov. 2008, vol. 38 , No.6, pp.1287--1290

[4] Rong-han Yao, Dian-hai Wang, Zhao-wei Qu. Equivalent queue lengthmodel for congested traffic stream based on two-fluid theory. JOURNAL OF SOUTHEAST UNIVERSITY(Natural Science Edition) ,May.2007, vol.37 ,No.3,pp.521--526

[5] Gartner NH, Messer C, Rathi A K. Monograph on Traffic Flow Theory [M].Washington: The Federal Highway Administration (FHWA), 1996.

[6] Dian-hai Wang, Chun-guang Jing, Zhao-wei Qu. Application of traffic-wave theory in intersections traffic flow analysis. China Journal of Highway and Transport,Jan.2002,vol.15,No.1,pp.93-96.

[7] Henry X. Liu, Xinkai Wu, Wenteng Ma, Heng Hu.Real-time queue length estimation for congested signalized intersections. Transportation Research Part C: Emerging Technologies.Volume 17, Issue 4, August 2009, Pages 412-427

[8] Dian-ha Wangi. Traffic Flow Theory [M]. Beijing: China Communications Press, 2002.

[9] Stephanopoulos and Michalopoulos, 1979.G. Stephanopoulos, P.G. Michalopoulos Modelling and analysis of traffic queue dynamics at signalized intersections Transportation Research (1979), p. 13A 